\title{
A Highly Conserved Effector in Fusarium oxysporum Is Required for Full Virulence on Arabidopsis
}

\author{
Louise F. Thatcher, Donald M. Gardiner, Kemal Kazan, and John M. Manners \\ CSIRO Plant Industry, Queensland Bioscience Precinct, St. Lucia, Queensland, 4067, Australia
}

Submitted 16 August 2011. Accepted 21 September 2011.

\begin{abstract}
Secreted-in-xylem (SIX) proteins of the vascular wilt pathogen Fusarium oxysporum f. sp. lycopersici are secreted during infection of tomato and function in virulence or avirulence. $F$. oxysporum formae speciales have specific host ranges but the roles of SIX proteins in diverse hosts are unknown. We identified homologs of $F$. oxysporum f. sp. lycopersici SIX1, SIX4, SIX8, and SIX9 in the genome of Arabidopsis infecting isolate Fo5176. A SIX4 homolog (termed Fo5176-SIX4) differed from that of $F$. oxysporum $f$. sp. lycopersici (Fol-SIX4) by only two amino acids, and its expression was induced during infection of Arabidopsis. Transgenic Arabidopsis plants constitutively expressing Fo5176-SIX4 had increased disease symptoms with Fo5176. Conversely, Fo5176-SIX4 gene knock-out mutants (Asix4) had significantly reduced virulence on Arabidopsis, and this was associated with reduced fungal biomass and host jasmonate-mediated gene expression, the latter known to be essential for host symptom development. Full virulence was restored by complementation of $\Delta$ six 4 mutants with either Fo5176-SIX4 or Fol-SIX4. Thus, Fo5176-SIX4 contributes quantitatively to virulence on Arabidopsis whereas, in tomato, Fol-SIX4 acts in host specificity as both an avirulence protein and a suppressor of other race-specific resistances. The strong sequence conservation for SIX4 in F. oxysporum f. sp. lycopersici and Fo5176 suggests a recent common origin.
\end{abstract}

To protect themselves from pathogens, plants employ multiple defenses acquired during plant-pathogen coevolution. A plant's first defensive front against microbial attack consists of nonspecific preformed physical and chemical barriers that restrict pathogen entry. In addition to these barriers, plants have an immune response that relies on the detection of generic pathogen-derived elicitors known as pathogen-associated molecular patterns (PAMPs) or microbe-associated molecular patterns (Bent and Mackey 2007; Boller and He 2009; Gohre and Robatzek 2008). Fungal cell wall components such as chitin, glucan, or glycoprotein and bacterial components such as lipopolysaccharide, elongation factor-Tu, or flagellin can all act as PAMPs. A PAMP-triggered defense response, termed PAMP-triggered immunity (PTI), usually halts or restricts the infection process; however, some microbes have adapted to this pressure by evolving effector proteins that interfere with or suppress the plant's defense response (Jones and Dangl

Corresponding author: L. Thatcher; Telephone +61 89333 6358; Fax +61 89387 8991; E-mail: Louise.Thatcher@csiro.au

* The $e$-Xtra logo stands for "electronic extra" and indicates three supplementary figures are published online.
2006). In response to this, plants have evolved specific receptors broadly referred to as resistance $(\mathrm{R})$ proteins that directly or indirectly recognize microbial effectors and trigger a second layer of defense termed effector-triggered immunity (ETI). Pathogens can avoid ETI and cause disease by eliminating or modifying the effector recognized by the plant's surveillance system. This co-evolutionary process between plants and microbial pathogens, originally proposed by Jones and Dangl (2006), has been reviewed extensively in recent years (Anderson et al. 2010; Bent and Mackey 2007; Boller and He 2009; Chisholm et al. 2006).

Much of our understanding of PTI and ETI has come from bacterial effectors (Gohre and Robatzek 2008). Like bacteria, fungal pathogens also secrete effectors, some of which have functions in virulence and others that are directly or indirectly recognized by $\mathrm{R}$ proteins and trigger immunity (Stergiopoulos and de Wit 2009). Usually, there is only a low degree of amino acid sequence conservation among fungal effectors across fungal lineages but most are thought to be secreted, cysteine-rich proteins. Although, potentially, a large number of effectors are produced during plant-fungus interactions, thus far, a function in virulence has only been determined for a few fungal effectors. For example, some effectors exhibit chitin-binding activity for protection from plant chitinases, or function as protease inhibitors (Stergiopoulos and de Wit 2009; Van De Wouw and Howlett 2011).

The fungal pathogen Fusarium oxysporum is responsible for vascular wilt disease, with pathogenic isolates classified into more than 120 formae speciales based on the individual plant species they infect. The infection process has been studied in detail in tomato (Michielse and Rep 2009) where, following germination of spores in the soil, hyphae colonize the root surface and directly penetrate the root epidermal layer, apparently at random sites, and advance through the root cortex intercellularly where they reach the xylem vessels. As a result of fungal colonization and host defenses attempting to limit pathogen spread, vessels become clogged, leading to water stress, collapse of vascular tissues, necrosis, and eventual plant death. Infection processes for strains of F. oxysporum infecting Arabidopsis differ slightly in that primary and lateral root initials appear to be a preferential site of initial fungal colonization and penetration (Czymmek et al. 2007; Kidd et al. 2011) and wilting and collapse of the foliar tissue is preceded by leaf chlorosis.

Four $R$ genes of tomato conditioning resistance against $F$. oxysporum f. sp. lycopersici have been identified in wild tomato relatives and introgressed into commercial tomato cultivars (Takken and Rep 2010). These are termed immunity (I) genes $I$, $I-I, I-2$, and $I-3$. Thus far, only $I-2$ has been cloned and encodes a nucleotide-binding leucine-rich repeat protein (Simons et al. 1998). Corresponding avirulence (Avr) genes in F. oxysporum $\mathrm{f}$. 
sp. lycopersici have been identified by reverse genetic analysis of genes encoding fungal proteins recovered from xylem sap of infected tomato plants, where they are termed secreted-in-xylem or (SIX) proteins (Rep et al. 2002, 2004; Takken and Rep 2010). SIX4 or Avr1 is the Avr gene product recognized by I and I-1. Similarly, SIX3 (also known as Avr2) and SIX1 (also known as Avr3) are recognized by I-2 and I-3, respectively. Interestingly, the tomato $R$ gene $I-2$ is expressed in cells associated with vascular tissues (Mes et al. 1999, 2000) where SIX proteins are secreted by the fungus. The cloning of the F. oxysporum avirulence SIX3/Avr2 (Houterman et al. 2009) along with the cognate $R$ gene (I-2) from tomato (Simons et al. 1998) represented the first complete $A v r-R$ gene pair molecularly characterized for a Fusarium pathogen and any root-infecting or xylem-colonizing fungus.

Some of the SIX proteins have been shown to have roles in both virulence and avirulence in a manner consistent with the PTI and ETI model for host-pathogen co-evolution (Jones and Dangl 2006) discussed above. Both SIX3/Avr2 (Houterman et al. 2009) and SIX1/Avr3 (Rep et al. 2004) are required for virulence on a fully susceptible tomato genotype. In contrast, SIX4/Avr1 appears not to be required for virulence on susceptible tomato and is dispensable and absent from the published F. oxysporum f. sp. lycopersici reference genome sequence (Ma et al. 2010). However, SIX4/Avr1 is required for virulence on tomato genotypes carrying either the $I-2$ or $I-3$ gene, where SIX4 appears to suppress their resistance function (Houterman et al. 2008). Therefore, it is believed that the acquisition of SIX4/Avrl has enabled F. oxysporum f. sp. lycopersici to overcome the resistance conferred by $I-2$ and $I-3$ without the penalty on virulence that would have resulted through the alternative of losing SIX3/Avr2 and SIXI/Avr3 (Takken and Rep 2010). The source of the SIX4/Avrl gene in F. oxysporum f. sp. lycopersici is not known but genome analysis of $F$. oxysporum f. sp. lycopersici has shown that other SIX effector genes are primarily encoded on supernumerary "pathogenicity-associated" chromosomes which can transfer between strains (Ma et al. 2010), facilitating such gene acquisition by horizontal chromosome transfer events (Mehrabi et al. 2011).

The Arabidopsis $-F$. oxysporum interaction is also developing as a model system to study host- $F$. oxysporum interactions, with some strains that cause disease on Brassica spp. also being able to infect Arabidopsis (Campbell et al. 2003; Diener and Ausubel 2005; Edgar et al. 2006). In some instances, these strains have been assigned to formae speciales including $F$. oxysporum f. sp. conglutinans, matthiolae, and raphani, which are pathogenic on cabbage, stock, and radish, respectively (Diener and Ausubel 2005). Most research on the Arabidopsis-F. oxysporum interaction has focused on the host reaction to infection and underpinning molecular mechanisms due to the large array of available genetic resources in Arabidopsis. Although the manipulation of key downstream signaling components such as hormone receptors, transcription factors, and defense genes can provide varying degrees of resistance to $F$. oxysporum in Arabidopsis (Anderson et al. 2004; Berrocal-Lobo and Molina 2004; Epple et al. 1997; Kidd et al. 2009; McGrath et al. 2005; Thatcher et al. 2009), no $R$ genes in a classical sense have been found. Variation in ecotype susceptibility and resistance has been identified (Czymmek et al. 2007; Diener and Ausubel 2005) (K. Kazan, unpublished) but, thus far, these differences appear polygenic in nature (Diener and Ausubel 2005). For example, six quantitative trait loci were identified in the Col-0 ecotype, with the single gene resistance to $\mathrm{F}$. oxysporum 1 (RFOl) being the largest contributor to resistance (Diener and Ausubel 2005). RFOl encodes a wall-associated kinase-like kinase and enhances resistance nonspecifically to several Brassica spp.-infecting F. oxysporum formae speciales.
In total, 11 SIX proteins have been identified thus far in $F$. oxysporum f. sp. lycopersici, and the function of most of these remains unknown (Houterman et al. 2007; Lievens et al. 2009; Ma et al. 2010). It has been shown that SIX genes are also present in formae speciales other than $F$. oxysporum $\mathrm{f}$. sp. lycopersici; for example, SIX6 and/or SIX7 homologs are present in F. oxysporum f. sp. lilii, melonis, radicis-cucumerinum, and vasinfectum (Chakrabarti et al. 2011; Lievens et al. 2009). However, the possible role in pathogenicity of SIX genes outside of $F$. oxysporum $\mathrm{f}$. sp. lycopersici has not been investigated. Assessments of SIX gene diversity and occurrence have been based on polymerase chain reaction (PCR) analysis, which may not always identify homologous sequences in other fungi. Using new short-read sequencing techniques, we sequenced the genome of the F. oxysporum isolate Fo5176 (Ma et al. 2010, supplementary data), which is virulent on Arabidopsis but does not cause disease on tomato (M. Rep, personal communication). Genome sequence mining facilitates the identification of $S I X$ gene homologs and, here, we report the presence of SIX1, SIX4, SIX8, and SIX9 homologs in the genome of the Arabidopsis infecting $F$. oxysporum isolate Fo5176. Even though the Fo5176 strain was isolated from infected Brassica oleracea in Australia and the strain Fol004 of F. oxysporum f. sp. lycopersici containing the SIX4/Avrl gene from infected tomato in the Netherlands (Mes et al. 1999; Rep et al. 2005), their SIX4 gene homologs are remarkably conserved and share more than $99 \%$ nucleotide and amino acid identity whereas other shared SIX genes have much weaker homologies. Furthermore, we show that full Fo5176 virulence on Arabidopsis requires the SIX4 gene, and the attenuated virulence in Fo5176-SIX4 knockouts can be complemented by the SIX4/Avrl gene of $F$. oxysporum f. sp. lycopersici. The SIX4 genes of $F$. oxysporum appear to contribute quantitatively to overall virulence on Arabidopsis but act qualitatively as either an $A v r$ gene or as a suppressor of other race-specific resistance in tomato. The high level of sequence conservation in SIX4 genes between strains infecting these diverse hosts suggests that functions in the two disparate hosts may be mechanistically related and that the genes probably share a recent common origin.

\section{RESULTS}

\section{A highly conserved $S I X 4$ homolog in Arabidopsis-infecting strain Fo5176.}

To determine if SIX genes are present in the Arabidopsisinfecting $F$. oxysporum isolate Fo5176, we queried a new 454 shotgun genome sequence assembly $(8 \times$ coverage, assembled into 7,858 contigs with a total genome size of $55 \mathrm{Mb}$ ) of Fo5176 with each of the available Fol-SIX gene sequences and identified putative homologs for SIX1,SIX4, SIX8, and SIX 9

Table 1. Percentage identity and similarity between Fusarium oxysporum f. sp. lycopersici secreted-in-xylem (SIX) and Fo5176-SIX homologues ${ }^{\mathrm{a}}$

\begin{tabular}{lccc}
\hline Fo5176-SIX & $\begin{array}{c}\text { GenBank } \\
\text { accession }\end{array}$ & $\begin{array}{c}\text { Amino acid } \\
\text { identity (\%) }\end{array}$ & $\begin{array}{c}\text { Amino acid } \\
\text { similarity (\%) }\end{array}$ \\
\hline SIX1 & HQ260601 & 74 & 80 \\
SIX4 & HQ260602 & 99.2 & 99.2 \\
SIX4b ${ }^{\text {b }}$ & HQ260605 & 81 & 89 \\
SIX8 & HQ260604 & 90 & 94 \\
SIX9 & HQ260603 & 53 & 66 \\
SIX9b & HQ260606 & 53 & 65 \\
\hline
\end{tabular}

a TBLASTN was used to identify Fol-SIX homologues in the Fo5176 genome.

b Available Fo5176-SIX4b sequence does not contain the N-terminal signal peptide; $5^{\prime}$ end of this sequence lies at the $5^{\prime}$ end of its contig. No overlapping contigs have been identified. 
(Table 1). Active genome sequencing of multiple $F$. oxysporum strains is in progress in several laboratories (L.-J. Ma, personal communication) and this will probably lead to many variants of SIX genes being identified. Therefore, we propose the following nomenclature to identify SIX gene homologs; [Fo] [forma speciales acronym if known] [strain code, if forma speciales is unknown or if there is variation within the forma speciales]-[SIX number]. For example, the original SIX4/AvrI (Houterman et al. 2007) would be termed Fol-SIX4 in this nomenclature because no sequence variants are known within $F$. oxysporum f. sp. lycopersici (M. Rep, personal communication).

Amino acid similarities between Fo5176-SIX1 and Fo5176SIX8 predicted proteins and their $F$. oxysporum f. sp. lycopersici homologs were 80 and 94\%, respectively, and two Fo5176-SIX9 proteins shared $66 \%$ (SIX9) and 65\% (SIX9b) similarity with Fol-SIX9. Fo5176-SIX4 was found on a 2,339-bp contig in the Fo5176 genome and this aligned completely at $99 \%$ nucleotide identity to the SIX4 region in Fol-SIX4. (Supplementary Fig. S1). Additional analysis identified another SIX4 homolog encoded on a distinct contig with $89 \%$ amino acid similarity with Fol-SIX4 (Table 1; Supplementary Fig. S2). Due to the higher level of conservation in the first SIX4 homolog, we herein call this Fo5176-SIX4 and call the second putative paralog Fo5176-SIX4b. From cDNA sequencing, the deduced Fo5176SIX4 coding sequence revealed $>99 \%$ amino acid identity with Fol-SIX4 and conservation of its single intron site and putative secretion signal sequence. Two single-nucleotide changes in exon two result in two amino acid differences in Fo5176-SIX4 compared with Fol-SIX4; an alanine (A) to glutamic acid (E) and glutamic acid (E) to glycine (G). The Fo5176-SIX4 and Fo5176-SIX4b open reading frames (ORF) share $77.2 \%$ nucleotide identity and, based on splice site prediction, Fo5176SIX $4 b$ also contains the conserved SIX4 intron. However, Fo5176-SIX4b appears to lack the N-terminal signal peptide but a possible start codon of Fo5176-SIX4b lies at the initial 5'

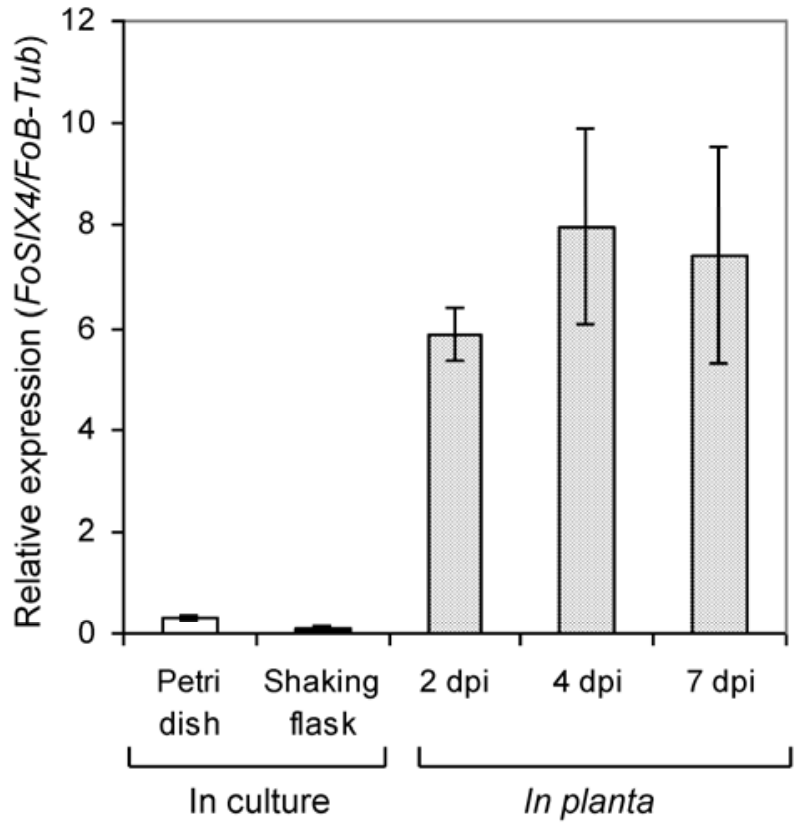

Fig. 1. Fo5176-SIX4 is highly expressed during infection of Arabidopsis roots. Fo5176-SIX4 expression was quantified in Fo5176 grown in potato dextrose broth and from infected Arabidopsis roots 2, 4, and 7 days postinoculation (dpi). Gene expression levels are relative to the internal control $\beta$-tubulin gene. Averages of three (petri dish, in planta) or four (shaking flask) biological replicates are shown with standard errors of the mean. Fo5176-SIX4 expression could not be detected in leaf tissue of infected plants at the time points sampled. end of its contig. Because an overlapping contig has not been identified, it is unclear whether a signal sequence may exist in SIX $4 b$.

The remarkable sequence conservation between Fo5176-SIX4 and Fol-SIX4 when compared with the sequence homologies in other SIX gene orthologs suggested that this gene may have an important function in $F$. oxysporum pathogenesis and, therefore, the role of SIX4 in the infection of Arabidopsis was studied further.

\section{Fo5176-SIX4 is highly expressed during Arabidopsis infection.}

SIX proteins were originally identified from the xylem sap proteome of $F$. oxysporum-infected tomato plants (Houterman et al. 2007; Rep et al. 2004). To determine whether Fo5176SIX4 homologs are expressed during infection of Arabidopsis, we quantified its mRNA expression (relative to that of fungal $\beta$-tubulin) in infected plants and axenic cultures using quantitative real-time PCR. No $\beta$-tubulin expression could be detected in leaves, indicating that Fusarium sp. growth had not progressed into these tissues at time of sampling. However, both $\beta$-tubulin and Fo5176-SIX4 expression were detected in root tissues where Fo5176-SIX4 was expressed at similar levels from 2, 4, and 7 days postinoculation (Fig. 1). Fo5176SIX4 expression was also detected in Fusarium spp. growing in potato dextrose broth (PDB); however, expression levels were 18- to 70-fold less than those measured in planta (Fig. 1), suggesting that its expression is highly responsive to the infection process. Compared with Fo5176-SIX4, the expression of Fo5176-SIX4b was 50- and $>100$-fold less in culture and in planta, respectively (data not shown), suggesting that Fo5176$S I X 4 b$ has little or no functional role in fungal pathogenesis, and this gene was not studied further.

\section{Fo5176 mutants lacking Fo5176-SIX4 have reduced} virulence and exhibit reduced growth within host tissue.

To examine the role of Fo5176-SIX4 in infection of Arabidopsis, we generated a Fo5176-SIX4 gene knockout construct by replacing 363 bp of Fo5176-SIX4 genomic sequence with the neomycin antibiotic resistance cassette (Fig. 2A). Introduction of this construct into Fo5176 through Agrobacterium sp.-mediated transformation generated two independent gene knockout mutants named $\Delta$ six4-1 and $\Delta$ six4-2 (Supplementary Fig. S3). Compared with Arabidopsis plants inoculated with Fo5176, $\Delta$ six4-1- and $\Delta$ six4-2-inoculated plants showed significantly reduced disease symptom development, with 71 and $42 \%$ less chlorosis at 7 days postinoculation and 66 and $28 \%$ less necrosis at 10 days postinoculation, respectively (Fig. 2B and C). The survival rates of $\Delta$ six4-inoculated plants were also significantly higher than Fo5176-inoculated plants, with 16 and 7 of 60 plants surviving ( $\Delta$ six $4-1$ and $\Delta$ six4-2, respectively) compared with only 1 of 60 plants for those inoculated with Fo5176 (Fig. 2D). Therefore, in contrast to Fol-SIX4, which is not required for full virulence on tomato (Houterman et al. 2008), our results demonstrated that Fo5176-SIX4 is required for full virulence on Arabidopsis. Growth rates and sporulation did not differ between Fo5176 and 4 six 4 mutants on agar plates or in broth (data not shown), suggesting that reduced virulence in $\Delta$ six4 was not due to slower fungal growth or development.

To determine whether fungal growth was reduced in plants inoculated with a $\Delta$ six 4 mutant, we quantified fungal biomass in the leaf tissue of plants inoculated with Fo5176 and the $\Delta$ six4-1 mutant in a separate experiment (Fig. 3). The reduced disease symptom development observed on $\Delta$ six4-1-inoculated plants (Fig. 2E) was associated with a significant reduction in fungal biomass, over 10-fold less than those inoculated with Fo5176 (Fig. 3). 


\section{Complementation of $\Delta$ six 4 mutants} with Fo5176-SIX4 restores full virulence.

To test that the reduced levels of virulence in $\Delta$ six4 mutants were due to deletion of Fo5176-SIX4, $\Delta$ six4 mutants were complemented with a Fo5176-SIX4 genomic sequence ( $\Delta$ six $4+$ Fo5176-SIX4) (Fig. 4). Following plant inoculation, both Fo5176-SIX4-complemented mutants had a restored full disease phenotype that was not significantly different in disease progression from inoculations with the wild-type fully virulent Fo5176 strain (Fig. 4B and C). Disease development with the complemented mutants and the Fo5176 strain were also both significantly higher than that observed with the two $\Delta$ six4 knock-out mutants (Fig. 4B).

\section{Fol-SIX4 complements reduced virulence} of $\Delta$ six 4 mutants on Arabidopsis.

As stated above, the Fol-SIX4 and Fo5176-SIX4 proteins differ by only 2 amino acids. Because Fol-SIX 4 has been reported not to be necessary for basal virulence on tomato, it was of interest to determine whether these amino acid changes played a role in virulence functions in Arabidopsis when compared with Fo5176-SIX4. When we introduced the Fol-SIX4

\section{A Deletion construct}

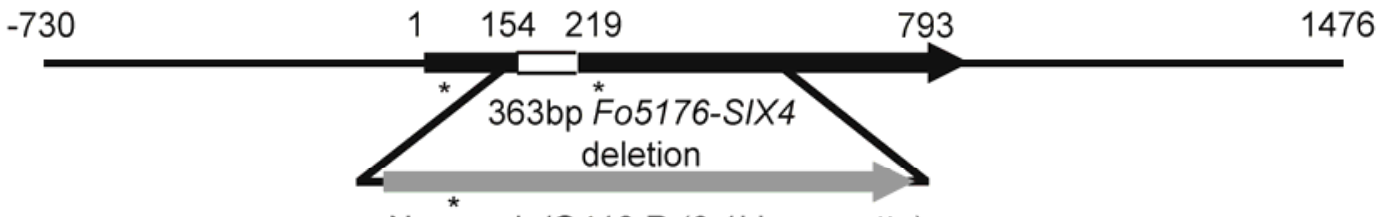

Neomycin/G418 R ( $3.1 \mathrm{~kb}$ cassette)
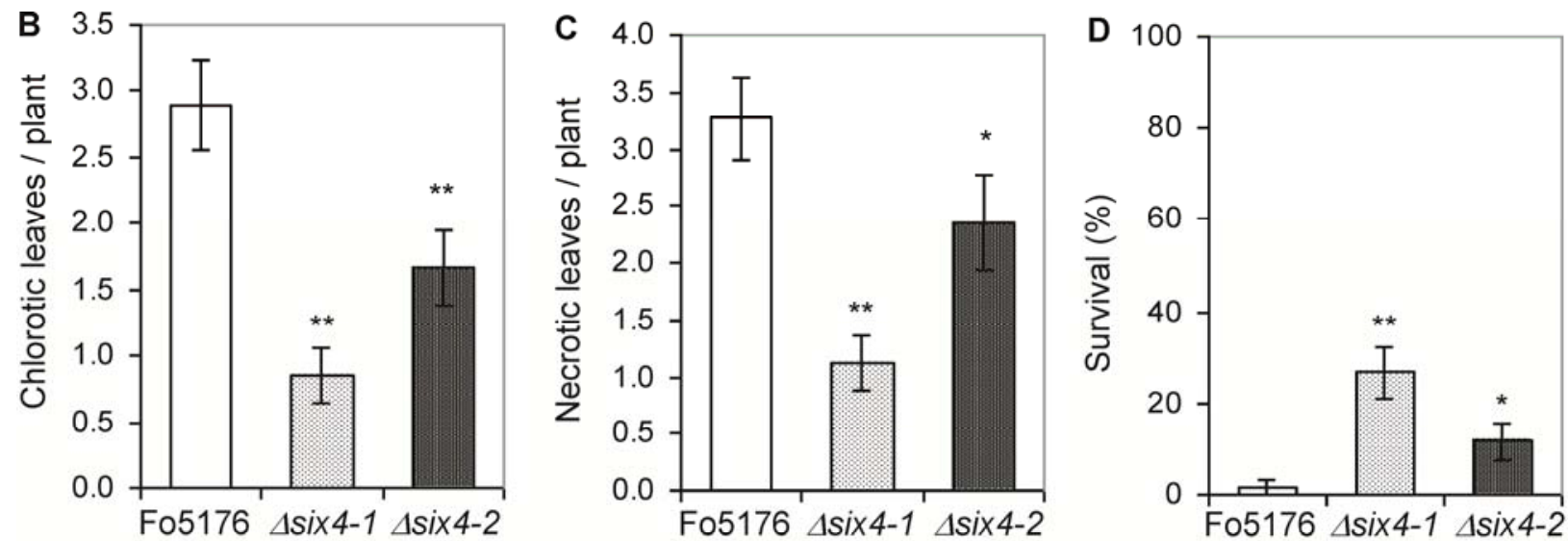

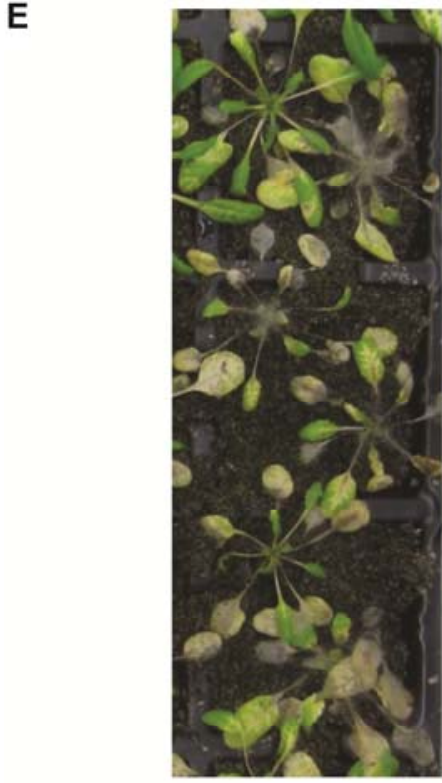

Fo5176

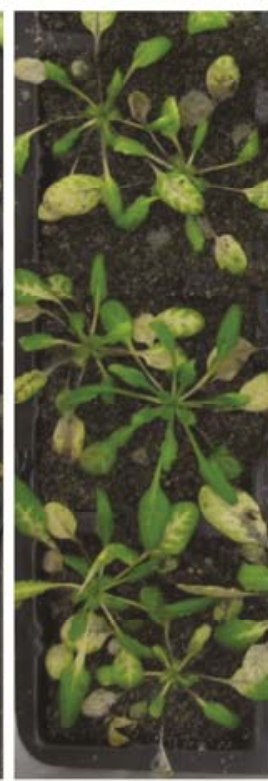

$4 \operatorname{six} 4-1$

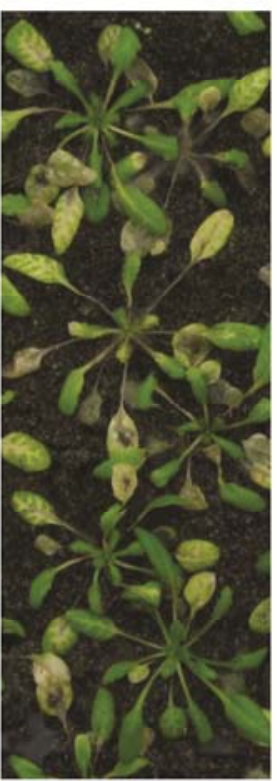

$4 \operatorname{six} 4-2$

Fig. 2. Fo5176 mutants lacking Fo5176-SIX4 have reduced virulence. A, The $\Delta$ six4 mutants were created using Agrobacterium sp.-mediated targeted deletion of the Fo5176-SIX4 gene. The location of the two secreted-in-xylem (SIX)4 exons (black boxes) and single intron (white box) are noted. Binding sites for primers used to confirm mutants are noted with an asterisk. B to E, Arabidopsis plants were inoculated with Fo5176 or two independent $\Delta$ six 4 mutants ( $\Delta$ six4-1 and $\Delta$ six4-2) and disease symptom development accessed. B, Chlorotic leaves/plant 7 days postinoculation; C, necrotic leaves/plant 10 days postinoculation; and $\mathbf{D}$, survival at 21 days postinoculation. The average of six replicates consisting of 10 plants each is shown with standard errors of the mean. Asterisks indicate values that are significantly different from Fo5176 (** $=P<0.01$ and $*=P<0.05$, Student's $t$ test). E, Disease phenotypes of plants 14 days postinoculation. Representative plants are shown. Similar results were obtained in independent experiments. 
gene into a $\Delta \operatorname{six} 4$ mutant, virulence was restored to the $\Delta \operatorname{six} 4$ mutants (Fig. 5A and B), suggesting that the 2-amino-acid difference between Fo5176-SIX4 and Fol-SIX4 is not specifically required for Fol5176-SIX4 virulence within the ArabidopsisF. oxysporum pathosystem. To test whether Fol-SIX4 might have a nonspecific effect on virulence, we also introduced FolSIX4 into wild-type Fo5176 but found no difference in disease symptom development between the two Fo5176 strains with and without Fol-SIX4 (Fig. 5C and D). These experiments confirm that Fol-SIX4 confers the same function as Fo5176SIX4 in disease symptom development on Arabidopsis.

Transgenic Arabidopsis plants expressing Fo5176-SIX4 have increased susceptibility.

Because plants inoculated with $\Delta$ six 4 had reduced disease symptoms, we were interested to determine whether expression of Fo5176-SIX4 in planta would cause increased susceptibility to Fo5176. Therefore, we generated Arabidopsis plants expressing the Fo5176-SIX4 coding sequence minus its predicted secretion signal under the control of the strong constitutive Cauliflower mosaic virus 35 S promoter (35S::Fo5176-SIX4). From independent T2 plant lines, we selected two homozygous lines with the highest Fo5176-SIX4 transcript expression for further analysis in T3 generation (Fig. 6A). Under normal growing conditions, these lines showed no obvious phenotypic differences from wild-type plants (data not shown). Upon inoculation with Fo5176, significantly more 35S::Fo5176-SIX4 plants developed disease symptoms than inoculated wild-type plants (Fig. 6B), suggesting that Fo5176-SIX4 promotes disease development, possibly by acting within host cells.

\section{Host jasmonate-mediated gene expression is reduced in $\Delta$ six 4 -inoculated plants.}

We have previously shown that host jasmonate (JA)-mediated gene expression is induced following $F$. oxysporum infection and that JA signaling promotes infection. For example, the

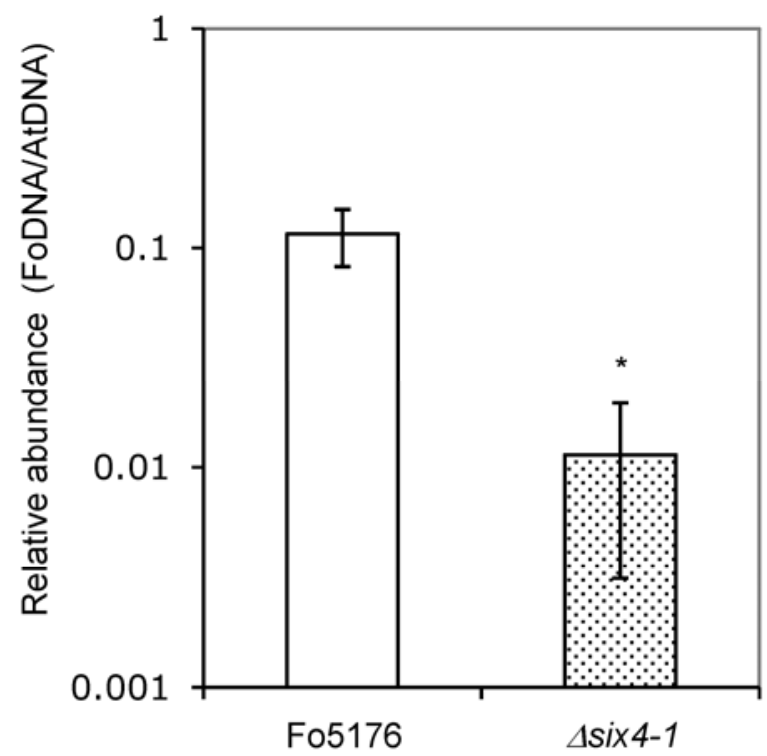

Fig. 3. Fungal growth is reduced in $\Delta$ six4-inoculated plants. Significantly less fungal biomass was detected in plants inoculated with $\Delta$ six $4-1$ compared with those inoculated with Fo5176. Values are relative to Arabidopsis DNA by assaying fungal glyceraldehyde 3-phosphate dehydrogenase (GPD; FOXG_08006) and Arabidopsis iASK (At5g26751) sequences from the leaves of inoculated plants by quantitative real-time polymerase chain reaction. The average of three independent replicates consisting of pools of six plants each is shown with standard errors of the mean. Asterisks indicate values that are significantly different $(*=P<0.05$, Student's $t$ test) from Fo5176.
Arabidopsis mutants coil and pftl both exhibit a high level of resistance to $F$. oxysporum and have reduced or almost absent activation of JA responses (Kidd et al. 2009; Thatcher et al. 2009). To determine whether the reduced disease symptom development on $\Delta$ six4-inoculated plants was associated with altered JA-mediated gene expression, we quantified the expression of JA biosynthesis ( $L O X 3$ and $O P R 3$ ), JA signaling (ERF1), and JA defense genes (PDF1.2, Thi2.1, and PR4) in Fo5176and $\Delta$ six4-inoculated plants (Fig. 7A to F). Compared with Fo5176 or ectopic control (contains both wild-type Fo5176SIX4 and knockout construct) F. oxysporum-inoculated plants, the expression of all JA-response genes tested showed reduced levels of expression in the $\Delta$ six4-inoculated plants, with reductions ranging from 1.5-fold ( $L O X 3)$ to 4.5 -fold (PDF1.2).

Because $\Delta$ six4-inoculated plants showed attenuated JAdefense gene expression, we also investigated whether salicylic acid (SA)-responsive defense genes showed any differential expression following inoculation. However, the expression of SA-marker genes pathogenesis-related $(P R) 1$ and $P R 2$ did not differ in plants inoculated with $\Delta$ six 4 , suggesting that decreased virulence of $\Delta \operatorname{six} 4$ was not due to upregulation of SA-mediated defenses (Fig. 7G and H).

\section{DISCUSSION}

Since the cloning of the first SIX gene from $F$. oxysporum $\mathrm{f}$. sp. lycopersici (Rep et al. 2002, 2004), 11 Fol-SIX effectors have been identified (Takken and Rep 2010). These small, cysteine-rich proteins are secreted into the xylem of infected tomato plants where roles in virulence or avirulence have been demonstrated for some (Houterman et al. 2007, 2008; Rep et al. 2004). Apart from a SIX1-related sequence in F. oxysporum f. sp. dianthi (Rep et al. 2004), homologs of SIXI to SIX5 have only been reported for $F$. oxysporum f. sp. lycopersici isolates (Lievens et al. 2009). Here, however, we identified homologs of SIXI and SIX4, along with SIX8 and SIX9 homologs, in an Arabidopsis-infecting $F$. oxysporum isolate. Although the Fo5176-SIX9 homologs shared only 53\% amino acid identity with that of $F$. oxysporum f. sp. lycopersici, Fo5176-SIX1 and Fo5176-SIX8 shared much higher identity with their $F$. oxysporum f. sp. lycopersici homologs (74 and 90\%, respectively). SIX6 and SIX7 homologs from $F$. oxysporum f. sp. lilii, melonis, and radicis-cucumerinum also share similar levels of amino acid identity with their F. oxysporum f. sp. lycopersici counterparts (Lievens et al. 2009). Strikingly, however, one of the Fo5176-SIX4 homologs we identified differed by only two nucleotides from Fol-SIX4 that result in 2 amino acid changes out of $242(0.8 \%$ at the amino acid level). The overall nucleotide identity with Fol-SIX4 across the entire 2,339-bp region of the Fo5176-SIX4 genome sequence contig was $99 \%$. Such a high level of $S I X$ gene conservation has previously been observed only within the same formae speciales among strains of similar host range (Houterman et al. 2009; Lievens et al. 2009; Rep et al. 2004). The presence of these two highly conserved effector genes in strains that are pathogenic on diverse hosts suggests that they either have a very recent common origin or have an evolutionarily conserved function that constrains gene diversification. The latter explanation seems less likely because the strong conservation of these genes extended outside of the coding region.

To dissect the function of Fo5176-SIX4 in the F. oxysporumArabidopsis interaction, we generated two independent $F$. oxysporum strains in which Fo5176-SIX4 had been deleted $(\Delta \operatorname{six} 4)$. Both $\Delta$ six 4 strains had significantly reduced virulence on Arabidopsis, and complementation of these mutants by reintroducing Fo5176-SIX4 restored the wild-type disease phenotype. Furthermore, we generated Arabidopsis plants express- 
ing Fo5176-SIX4 and these plants exhibited increased disease symptom development upon $F$. oxysporum infection (Fig. 6). Fol-SIX4, however, is not required for full virulence on tomato because its removal has no impact on the disease phenotype of susceptible plants that contain no $I$ genes (Houterman et al. 2008). In F. oxysporum f. sp. lycopersici-tomato interactions, Fol-SIX4 acts as an Avr protein where it triggers resistance in $I$ - or $I$ - 1 -containing tomato and as a suppressor of resistance in $I-2$ or $I-3$ lines (Houterman et al. 2008). How Fol-SIX4 suppresses resistance is not known; however, it is suggested that it may interfere with either uptake from the apoplast of Fol-SIX1 and Fol-SIX3 or I-2 and I-3 functions, or signal transduction downstream of these $\mathrm{R}$ proteins (Houterman et al. 2008). In addition, all race $1 F$. oxysporum $\mathrm{f}$. sp. lycopersici strains con- tain SIX4 along with the virulence effectors SIX1 and SIX3 (Houterman et al. 2008; Lievens et al. 2009; Rep et al. 2005; Takken and Rep 2010). It has been proposed that Fol-SIX4 has probably been acquired and retained because of its ability to suppress I-2 and I-3 resistances and that the $I$ and $I-1$ resistance genes evolved later to recognize Fol-SIX4 as an Avr protein (Houterman et al. 2008). Major gene resistance to specific races of $F$. oxysporum is not known in Arabidopsis, where only polygenic resistance has been described (Diener and Ausubel 2005). Therefore, it is unlikely that the SIX4 virulence function demonstrated here works by suppression of a specific resistance gene. However, it is still possible that it may act on processes common to both tomato and Arabidopsis such as mechanisms of defense-related signal transduction.

\section{A Complementation construct}

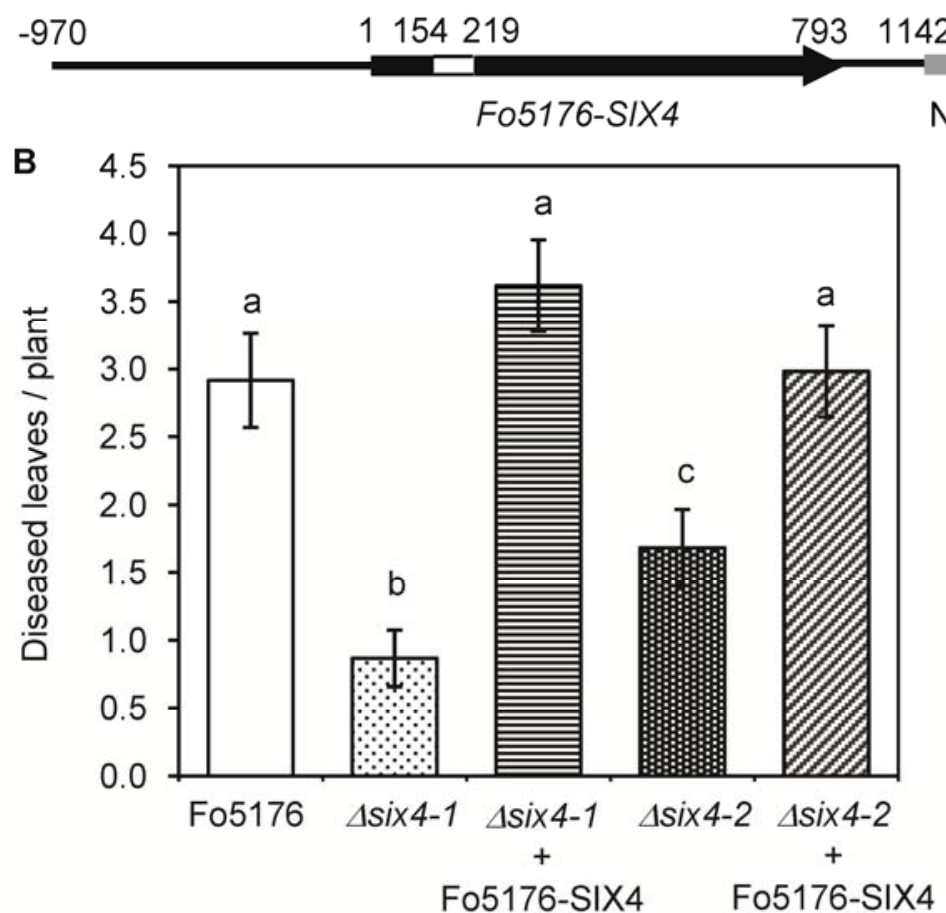

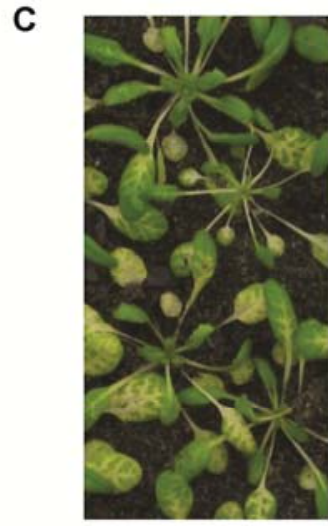

Fo5176

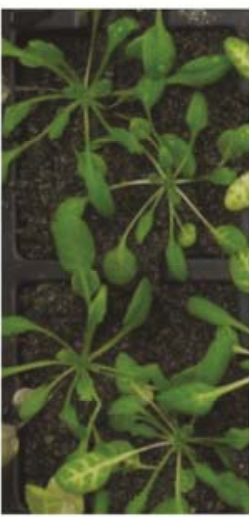

$\Delta \operatorname{six} 4-1$

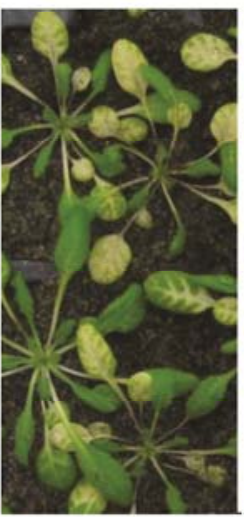

$\Delta \operatorname{six} 4-1$

$+$

Fo5176-SIX4

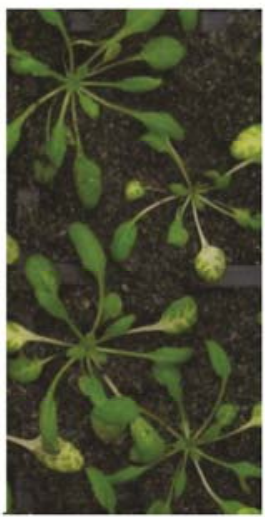

$\Delta s i x 4-2$

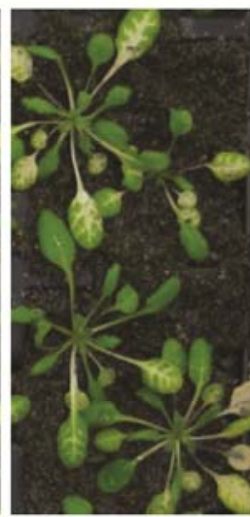

$\Delta s i x 4-2$

$+$

Fo5176-SIX4

Fig. 4. Fo5176-SIX4 complementation restores full virulence in $\Delta$ six 4 mutants. A, The $\Delta$ six 4 mutants were complemented ( $\Delta$ six $4+$ Fo5176-SIX4) by transformation of $\Delta$ six 4 with -970 to 1,142 bp of the Fo5176-SIX4 gene. The location of the two secreted-in-xylem (SIX) 4 exons (black boxes) and single intron (white box) are noted. B, Diseased leaves/plant 7 days postinoculation. The average of six replicates consisting of 10 plants each is shown with standard error. Letters indicate values that are significantly different from each other $(P<0.05$, all pairs Student's $t$ test $)$. C, Disease phenotypes of plants inoculated 7 days postinoculation. Representative plants are shown. Similar results were obtained in independent experiments. 
The requirement of Fo5176-SIX4 for full Fo5176 virulence on Arabidopsis has led us to examine its mode of action in further detail. First, although Fo5176-SIX4 expression was detectable in culture, its expression increased greatly upon infection of Arabidopsis roots (Fig. 1). Fo5176-SIX4 expression in infected roots was up to 70 times higher than in culture, suggesting that it is highly responsive to infection cues. A similar expression pattern occurs for Fol-SIXI during infection of tomato (Rep et al. 2004; van der Does et al. 2008). Fol-SIX1 expression requires host cells and is highly expressed in root tissues. The lack of or very low levels of Fol-SIX1 and Fo5176-SIX4 expression in the absence of a host suggest that SIX effector genes are induced specifically during host colonization. It has been suggested by van der Does and associates (2008) that this ability may be used by $F$. oxysporum to switch between its biotrophic invading phase and its necrotrophic phase, where SIX effectors may not be necessary.

In tomato xylem sap, several PR proteins have been detected specifically upon $F$. oxysporum infection. These include members upregulated through SA- and JA-signaling pathways such as PR1, glucanase (PR2), chitinase (PR3 and PR4), PR5, and several peroxidases (Houterman et al. 2007; Rep et al. 2002). In the Arabidopsis-Fusarium pathosystem, $P R$ genes regulated through the SA pathway are either suppressed or nonresponsive to infection (Edgar et al. 2006; Kidd et al. 2011), suggesting that defense responses mediated by SA are not activated during infection. Indeed, genetic studies and hormone treatments indicate only a minor effect of SA on Fusarium wilt disease outcome in Arabidopsis (Diener and Ausubel 2005; Edgar et al. 2006; Thatcher et al. 2009). In contrast, we have found that the JA pathway plays a major role in disease development in Arabidopsis. For example, the high level of $F$. oxysporum resistance in the jinl/myc2 mutant is likely mediated, at least in part, by its specific upregulation of JA-mediated defenses (Anderson et al. 2004), while resistance in coil and pftl mutants is likely mediated by their overall reduction in nondefensive JA responses such as JA-induced senescence (Kidd et al. 2009; Thatcher et al. 2009). A common attribute of these plant mutants is their reduced sensitivity to JA; therefore, we have proposed that $F$. oxysporum hijacks aspects of the JA-signaling pathway to promote wilt-disease symptoms that eventuate in plant death. Interestingly, the reduced disease symptom development in plants inoculated with Fo5176 lacking SIX4 ( $\Delta$ six4) was associated with a significant reduction in the expression of JA-defense, -biosynthesis, and -signaling genes, whereas there was no difference in SA-marker gene expression (Fig. 7). This sug-
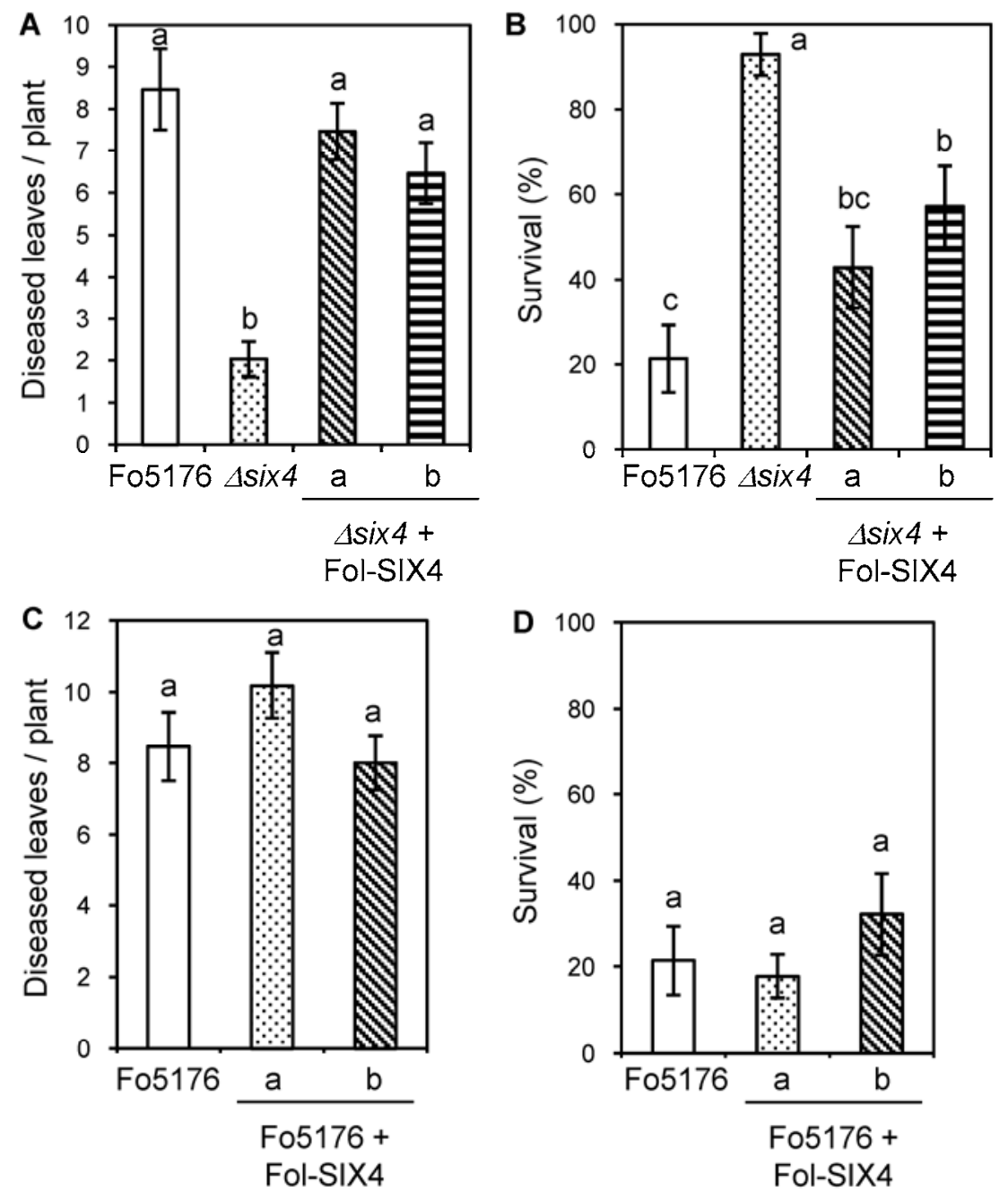

Fig. 5. Fol-SIX4 complements $\Delta$ six 4 virulence but does not affect virulence or avirulence in wild-type Fusarium oxysporum Fo5176. A and B, Mutant $\Delta$ six 4 was complemented ( $\Delta$ six $4+$ Fol-SIX4) by transformation of $\Delta$ six 4 with -708 to 1,142 bp of the Fol-SIX4 gene. C and D, Fo5176 transformed with -708 to 1,142 bp of the Fol-SIX4 gene (Fo5176 + Fol-SIX4). A and C, Diseased leaves/plant 14 days postinoculation. B and D, Survival at 21 days postinoculation. The $\Delta$ six $4+$ Fol-SIX4 and Fo5176 + Fol-SIX4 lines a and b represent independent transformants. The average of four replicates consisting of seven plants each is shown with standard errors of the mean. Letters indicate values that are significantly different from each other $(P<0.05$, all pairs Student's $t$ test). Similar results were obtained in independent experiments. 
gested that the virulence activity of Fo5176-SIX4 may function through activation of host JA responses. Bacterial effectors unrelated to SIX4 proteins that promote jasmonate signaling to induce host susceptibility have been identified (Cui et al. 2010). To determine whether Fo5176-SIX4 itself could induce or prime host JA responses in the absence of other Fusarium sp. elicitors, we also examined gene expression in 35S::Fo5176-SIX4 transgenic Arabidopsis plants. With the exception of the JA-repressor gene $J A Z 10$, we were unable to observe consistent and uniform upregulation of JA-responsive genes in both of the $35 S:: F o 5176$ SIX4 lines (data not shown). This suggests that Fo5176-SIX4 is unable to induce host JA responses on its own.

The finding that Fo5176-SIX4 did not activate host JA responses when transgenically expressed from the host genome, at least on its own, but did cause increased disease symptom development upon infection suggests that Fo5176-SIX4 either acts through other pathways to enhance virulence or requires other Fusarium effectors or elicitors to participate in the hijacking of host JA signaling. If Fo5176-SIX4 acts via other virulence mechanisms, then the reduced JA-associated gene expression following infection by $\Delta$ six 4 was most probably a consequence of much less fungal biomass being produced by $\Delta$ six 4 than the Fo5176 wild-type and, therefore, reduced colonization and less ability to stimulate the JA pathway (Fig. 3). The SIX4 protein of $F$. oxysporum $\mathrm{f}$. sp. lycopersici is known to be a secreted protein during colonization of tomato (Houterman et al. 2007), and complete conservation of the signal peptide between Fol-SIX4 and Fo5176-SIX4 indicates that Fo5176-SIX4 is also secreted. Our plant transformation construct was designed to express the Fo5176-SIX4 protein without its signal peptide directly in the plant cytoplasm. The enhanced susceptibility of plants expressing the putative mature Fo5176-SIX4 protein is consistent with it having a role in virulence and also that its site of action is within the host cell cytoplasm. Fol-SIX3 is also recognized intracellularly, implying uptake of SIX proteins by host cells (Houterman et al. 2009). Our experiments, however, do not rule out the possibility that Fo5176-SIX4 may also have a virulence function in the apoplast.

Several copies of SIX genes have previously been observed in F. oxysporum f. sp. lycopersici isolates, with several containing two SIX1 genes (Rep et al. 2004) and several copies of
SIX8 existing in Fol4287 (M. Rep and L. J. Ma, unpublished). We also identified a second Fo5176-SIX4 gene and two Fo5176-SIX9 homologs. Although we found that Fol-SIX4 fully complemented the virulence function of Fo5176-SIX4 (Fig. 5), the 2-amino-acid changes between SIX4 from $F$. oxysporum f. sp. lycopersici and Fo5176 may affect its recognition within the host. Indeed, a single nucleotide change in FolSIX3 affects its recognition by the R protein $\mathrm{I}-2$ but not its virulence on tomato plants lacking I-2 (Houterman et al. 2009). Our finding that addition of Fol-SIX4 to wild-type Fo5176 did not affect disease symptom progression (Fig. 5C and D) demonstrates that the 2-amino-acid differences between Fo5176-SIX4 and Fol-SIX4 are functionally benign and do not confer either added virulence or avirulence functions to Fol-SIX4 on Arabidopsis.

With the exception of Fol-SIX8, which is present on multiple chromosomes, all Fol-SIX genes in the sequenced race 2 strain Fol4287 are located on chromosome-14 that is supernumerary within F. oxysporum f. sp. lycopersici (Ma et al. 2010). Furthermore, Ma and associates (2010) demonstrated that chromosome 14 of $F$. oxysporum f. sp. lycopersici could transfer vegetatively to otherwise genetically isolated strains and confer virulence on tomato, suggesting that effector genes may evolve independently of the basal genome on supernumerary mobile chromosomes. Fol-SIX4 is absent from the sequenced reference strain Fol4287 and all other race 2 strains; however, recent analysis of diverse $F$. oxysporum $\mathrm{f}$. sp. lycopersici strains has demonstrated that Fol-SIX4 is not located on chromosome 14 but on another putative supernumerary chromosome (M. Rep, personal communication). Thus, the acquisition of SIX4 by strains of $F$. oxysporum f. sp. lycopersici and Fo5176 was also probably a result of a horizontal chromosome transfer event. It is likely that SIX genes located on distinct supernumerary chromosomes may be mobilized and evolve independently, and that separate chromosomal origins, compared with that observed between the other SIX genes, may explain the substantial sequence conservation of Fo5176-SIX4 with FolSIX4. The high level of Fol-SIX4 and Fo5176-SIX4 gene conservation suggests that SIX 4 transfer occurred recently and was probably from a related source. Because the SIX4 gene appears to play a critical role in $F$. oxysporum virulence on multiple
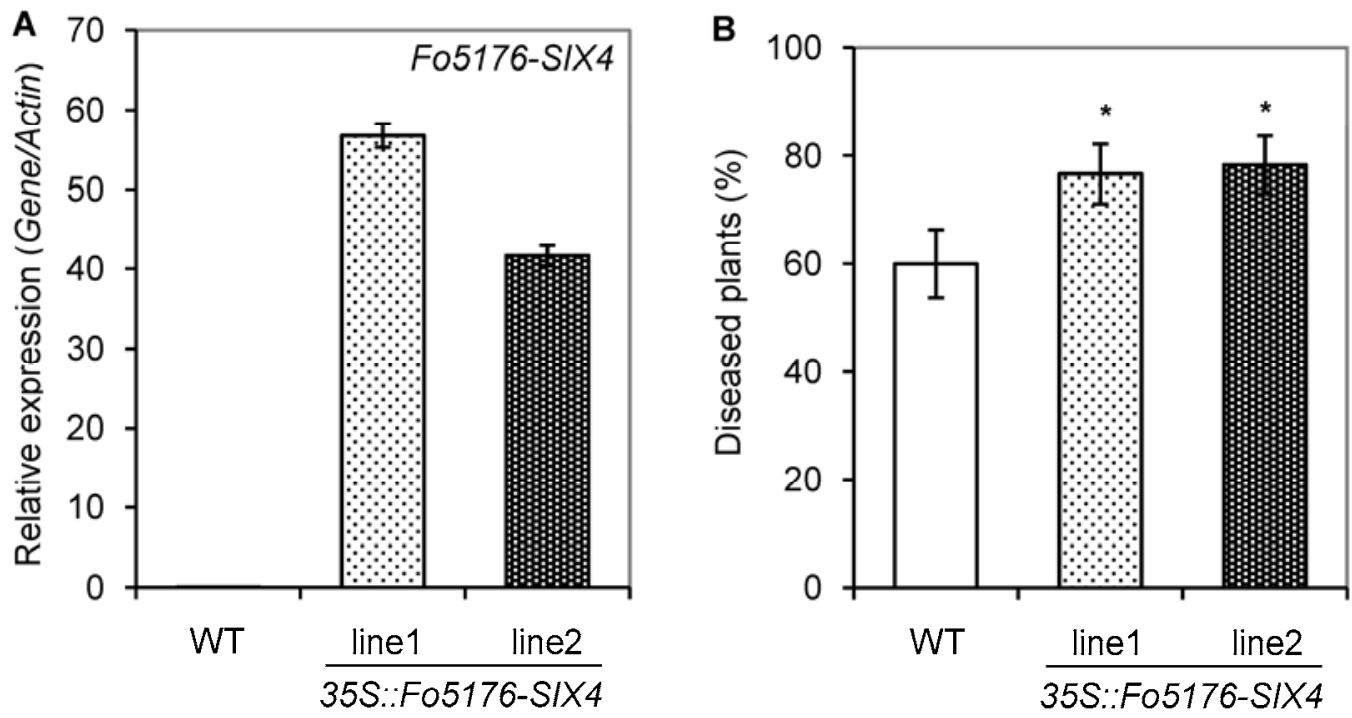

Fig. 6. Arabidopsis plants expressing Fo5176-SIX4 are more susceptible to Fusarium disease symptom development. Wild-type (WT) and Fo5176-SIX4expressing (35S::Fo5176-SIX4) plants were inoculated with Fo5176. A, Prior to fungal inoculation, Fo5176-SIX4 expression was measured in planta. B, Diseased plants at 21 days postinoculation. Averages of three replicates (gene expression) or six replicates (disease phenotypes) consisting of pools of 10 plants are shown with standard errors of the mean. Asterisks indicate values that are significantly different $(*=P<0.05$, Student's $t$ test) from the WT. Similar results were obtained in independent experiments. 
hosts, its molecular mode of action warrants further investigation.

\section{MATERIALS AND METHODS}

SIX gene identification and sequence analysis.

The F. oxysporum f. sp. lycopersici SIX protein sequences were obtained from GenBank accessions ACY39281 to ACY39287 and ACN69118, and from the Broad Fusarium website, FOXG_14223 and FOXG_17457. Fo5176 genomic sequence was previously obtained using Illumina sequencing (Ma et al. 2010) but additional sequencing was performed here using 454 technology. The 454 sequencing was performed using GS-FLX Titanium chemistry on a shotgun library sequenced across two plates. Sequencing and de novo assembly were performed by the Australian Genome Research Facility. The genome assembly consisted of 7,858 contigs greater than 199 bp in size, with an average coverage eightfold and an N50 of $60 \mathrm{~kb}$. The genome was annotated for gene content using FGENESH (Salamov and Solovyev 2000) as part of the MolQuest2 package. This Whole Genome Shotgun project has been deposited at DDBJ/EMBL/GenBank databases under the accession AFQF00000000. The version described in this article is the first version, AFQF01000000.

In-house TBLASTN (Altschul et al. 1997; Altschul et al. 2005) against the consensus contig sequences was used to identify Fo5176-SIX homologs in the Fo5176 genome. Contigs containing SIX homologs were extracted and, for SIX8 and
SIX9 homologs, gene structure was predicted using homology to $F$. oxysporum f. sp. lycopersici SIX proteins with FGENESH+ (Softberry, Mount Kisco, NY, U.S.A.), because these genes had been missed altogether or incorrectly predicted with the initial $a b$ initio prediction. After manual removal of a small number of very short incomplete gene predictions, the final gene number was 17,817. Locus numbers are in the format FOXB_XXXXX. Fo5176-SIX gene homologs are available at GenBank (HQ260601 to HQ260606). Amino acid and nucleotide alignments were performed with ClustalW and Align (available on the world wide web).

\section{Quantitative reverse-transcription (Q-RT)-PCR.}

For F. oxysporum gene expression, Q-RT-PCR experiments were performed from tissue grown in PDB or harvested from infected Arabidopsis roots. For stationary cultures, $100 \mu \mathrm{l}$ of $1 \times$ $10^{6} \mathrm{spores} / \mathrm{ml}$ were transferred into a petri dish containing 50 $\mathrm{ml}$ of PDB and incubated at room temperature. For the shaking cultures, a flask of PDB was inoculated with an $F$. oxysporum agar plug. After 3 days, the resulting mycelia and spores were harvested through Miracloth (Calbiochem, San Diego, CA, U.S.A.), rinsed with sterile water, and frozen in liquid nitrogen. Three biological replicates were taken for stationary cultures and infected root samples and four for shaking flask samples. RNA extraction, cDNA synthesis, and Q-RT-PCR were conducted as described by McGrath and associates (2005) using an Applied Biosystems 7900HT Fast Real-Time PCR System (Foster City, CA, U.S.A.). Absolute gene expression levels
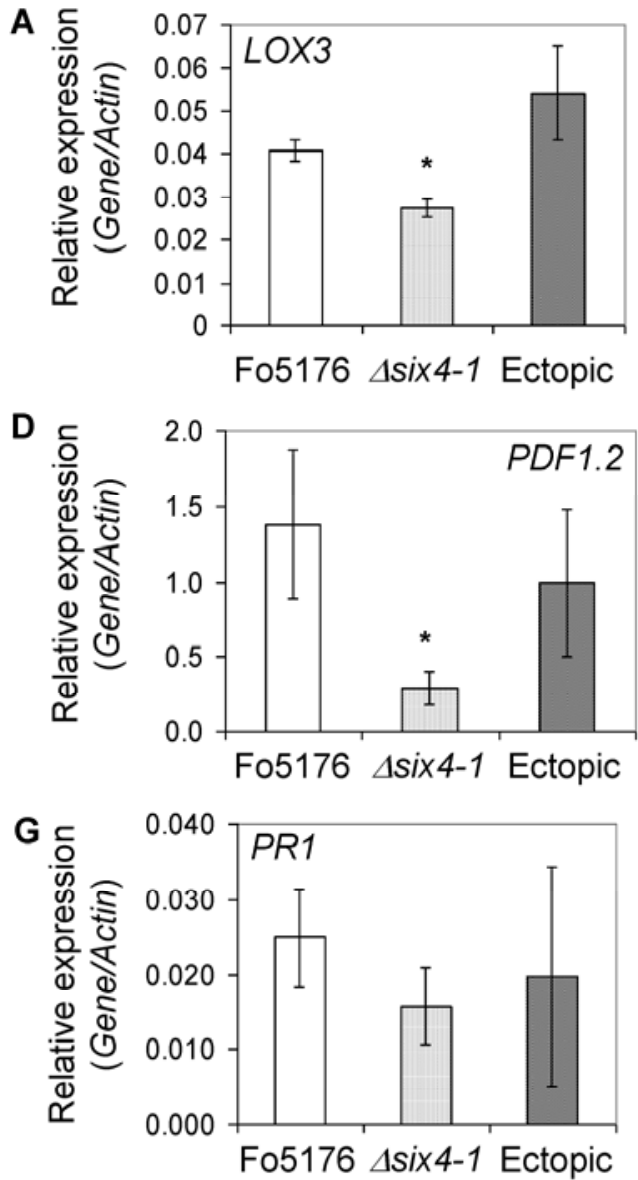

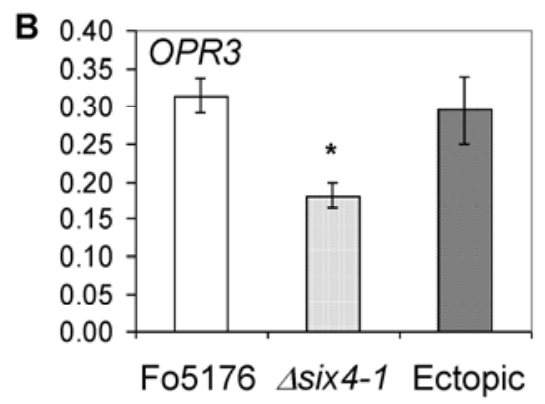

E
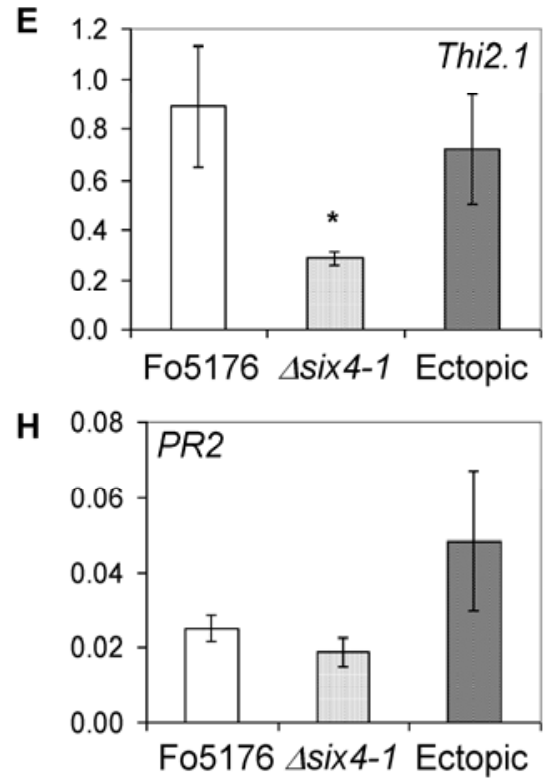
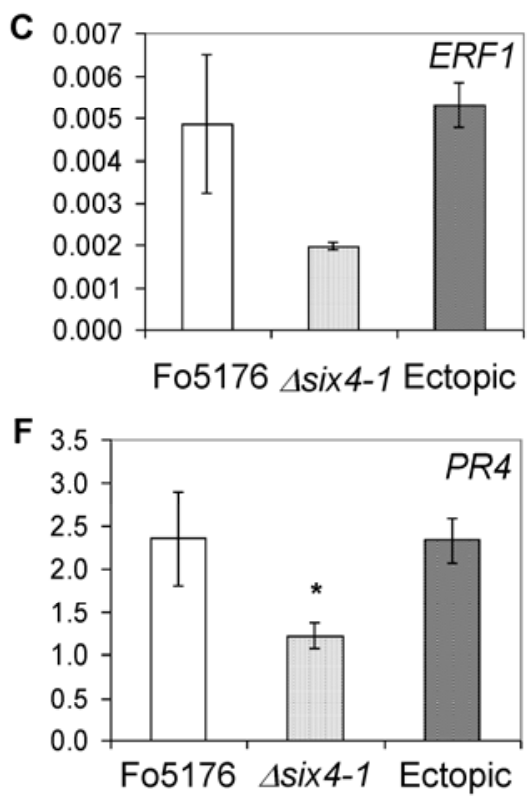

Fig. 7. Host jasmonate (JA)-mediated gene expression is reduced in $\Delta$ six4-inoculated plants. Expression of $\mathbf{A}$ and $\mathbf{B}$, JA biosynthesis; C, the positive JA regulator ERF1; and $\mathbf{D}$ to $\mathbf{F}$, JA-mediated defense gene expression is reduced in plants inoculated with $\Delta$ six4. $\mathbf{G}$ and $\mathbf{H}$, Reduced activation of JA-mediated gene expression does not correlate with increased salicylic-acid-mediated gene expression. The average of three independent replicates consisting of pools of 10 plants each is shown with standard error. Asterisks indicate values that are significantly different $(*=P<0.05$, Student's $t$ test) from Fo5176. An ectopic strain was used as a Fusarium transformation control and contains the wild-type Fo5176-SIX4 gene along with the knockout construct. 
relative to the $\beta$-tubulin reference gene were used for each cDNA sample using the equation: relative ratio gene of interest/ $\beta$-Tub $=\left(\right.$ Egene $\left.^{-\mathrm{Ct} \text { gene }}\right) /\left(\mathrm{E} \beta-\mathrm{Tub}^{-\mathrm{Ct} \beta-\mathrm{Tub}}\right) . \beta$-Tubulin primers were designed from FOXG_06228 and were Fo $\beta$-Tub-F 5'-TGTTC GACCCCAAGAACAT-3' and Fo $\beta$-Tub-R 5'-GGTCCTCGAC CTCCTTCATA-3'. Fo5176-SIX4 primers were designed from the $F$. oxysporum f. sp. lycopersici sequence AM234064 and were SIX4RT-F 5'-GACGGGCATTATGGACAAAC-3' and SIX4RT-R 5'-CGCTGGAAGGATCAAAGTTC-3'.

For Arabidopsis gene expression, Q-RT-PCR experiments were performed on shoot tissue collected before or 4 days after $F$. oxysporum inoculation. Three biological replicates were taken, consisting of tissue pooled from 5 to 10 plants. Plants were cut at the top of the root, just below the crown; therefore, shoot tissue consisted of the hypocotyl and aerial tissue. Absolute gene expression levels relative to the previously validated reference genes $\beta$-actin $2, \beta$-actin 7 , and $\beta$-actin 8 (At1g49240, At3g18780, and At5g09810, respectively) were used for each cDNA using $\left(\right.$ Egene $^{-\mathrm{Ct}}$ gene $) /\left(\right.$ Eactin $\left.^{-\mathrm{Ct} \text { actin }}\right)$. The gene-specific primer sequences have been previously published (Anderson et al. 2004; Edgar et al. 2006; Thatcher et al. 2009).

\section{Fo5176-SIX4 disruption and complementation.}

Approximately $2.2 \mathrm{~kb}$ of Fo5176-SIX4 spanning from -730 bp (promoter relative to start codon) to 1,476 bp (ORF and 3' untranslated region [UTR]) was amplified off genomic DNA and cloned into pPZP200 in DY380 Escherichia coli. (Fo_SIX4 -730PstI-F 5'-GGTCTGCAGACACACTGGTCGACTTAGA3' Fo_SIX4 3'UTRKpnI-R 5'-GGCGGTACCATCGAGAAGT GGTGGTCAA-3'). The neomycin cassette for insertion into FoSIX4-pPZP200 was amplified from pAN9.1 using Fo_SIX4 NeoRBM-F 5'-GGGTGACATCATCGGAACATTCAACTTT AGCAGCTCAGACAGTCAAGAAGAACTGGAGGGGTG-3' and Fo_SIX4 NeoRBM-R 5'-CATGTGTTGCCTCCTTGTCA TCTACCGCATCAAAAACAGTGTTAGTAACCTGAGGCTA TGGCA- $3^{\prime}$ and then recombined according to Gardiner and associates (2009). The resulting plasmid, FoSIX4-NeopPZP200, was transformed into DH10 E. coli to remove any nonrecombined plasmid. FoSIX4-Neo-pPZP200 was then transformed into Agrobacterium AGL1 and was used to transform Fo5176 according to Kidd and associates (2011). Transformants were selected on potato dextrose agar (PDA) with G418 (Sigma-Aldrich, St Louis) at $50 \mathrm{mg} /$ liter and confirmed by PCR from individual DNA extractions using SIX4-F (5'GTTATTGCTTCGGTGGCTGT-3') and SIX4-del-R (5'-CAG ACTCGGTATGAGCAGAT-3') (for absence of wild-type band) and SIX4-F to Neo-R (5'-ACGTTTGACTTGCATTGTGC-3') (for presence of neomycin cassette). The $\Delta$ six 4 knockouts were purified via single-spore isolation, confirmed by PCR, and stored dry on filter paper at $4^{\circ} \mathrm{C}$.

Complementation of $\Delta$ six 4 was achieved by transformation with $-970 \mathrm{bp}$ (promoter relative to start codon) to $1,142 \mathrm{bp}$ of Fo5176-SIX4 genomic sequence using Fo_SIX4 -970 BamHI-F 5'-CTCGGATCCAAAGAAGGTTATTACCGAT-3' and Fo_SIX4 +1,142 MfeI-R 5'-CGGCAATTGCGGTCAAGA AATTAAAGAT-3'. The resulting amplicon was cloned into BamHI/EcoRI-digested pPZPnat1 (Gardiner et al. 2005) to produce FoSIX4-comp-pPZPnat1, moved into Agrobacterium AGL1, and transformed into $\Delta$ six 4 to produce $\Delta s i x 4+F o 5176$ SIX4. Transformants were selected on PDA with nourseothricin (Werner BioAgents, Jena, Germany) at $50 \mathrm{mg} / \mathrm{liter}$ and confirmed by PCR from individual DNA extractions using SIX4-F and SIX4 del-R (for presence of the wild-type band). Two complements for each $\Delta$ six 4 strain were single spored, confirmed by PCR, and stored on filter paper.

Complementation of $\Delta$ six 4 or Fo5176 with Fol-SIX4 was achieved by amplification of -708 to 1,142 of Fol-SIX4 from the vector SIX4 pRW1p (Houterman et al. 2008) using Fol_SIX4 -708 BamHI-F 5'-CGAGGATCCGTTTACTCCGC CTGGCTAC-3' and Fo_SIX4 +1,142 MfeI-R. The amplicon was cloned into pPZPnat1 and moved into Agrobacterium. Transformants were selected and confirmed as above.

\section{Plant material and growth conditions.}

Unless otherwise specified, all experiments were conducted with the Arabidopsis thaliana Columbia-0 (Col-0) ecotype grown in soil under a short-daylight regime $(8 \mathrm{~h}$ of light and $16 \mathrm{~h}$ of darkness) at $21^{\circ} \mathrm{C}$ as described previously (Campbell et al. 2003; Edgar et al. 2006). To clone the Fo5176-SIX4 coding sequence (CDS) for its expression in Arabidopsis, total RNA was isolated from mycelia grown in PDB and used to generate cDNA as described above for Q-RT-PCR. For generation of plants expressing the Fo5176-SIX4 CDS minus the signal peptide (35S::Fo5176-SIX4), the Fo5176-SIX4 CDS was amplified using Fo_SIX4 HindIII-F 5'-TGGCTAAGCTTAACAATGCT TCCAAAGGGGGAG-3' which includes an ATG start codon, and Fo_SIX4 EcoRI-R 5'-CTTAGAATTCCTAAGCTAAGTT AAGTGT-3'. Removal of the signal peptide results in a mature SIX4 protein starting with the amino acids MLPKGEE. The resulting amplicon was cloned into HindIII/EcoRI-digested binary vector pKEN (McGrath et al. 2005) and confirmed by sequencing. 35S::Fo5176-SIX4 pKEN was mobilized into Agrobacterium AGL1 and transformed into A. thaliana as per McGrath and associates (2005). Transgenic plants were selected based on their resistance to Pestanal (glufosinate-ammonium; Riedel-de Haen, Seelze, Germany) at $10 \mathrm{mg} / \mathrm{liter}$ and resulting T3 lines were used in subsequent experiments.

\section{F. oxysporum inoculation.}

The $F$. oxysporum isolate used in this study was strain Fo5176 obtained from R. Shivas, Queensland Plant Pathology Herbarium, Queensland Department of Primary Industries and Fisheries, Brisbane, Australia. Inoculations were performed as described by Anderson and associates (2004) and Edgar and associates (2006).

\section{Fungal biomass estimation.}

Fungal biomass measurements were performed as described previously (Thatcher et al. 2009) with whole aboveground tissues for DNA extractions collected at 10 days postinoculation.

\section{F. oxysporum growth assay.}

Strains $\Delta$ six4 and its complement $\Delta$ six4 + Fo5176-SIX4 were grown on an appropriate antibiotic selection and then an infected agar plug transferred to media without selection (PDA). The radius was measured daily for 7 days and the growth rate per day (in millimeters) was determined.

\section{ACKNOWLEDGMENTS}

L. F. Thatcher is supported by a CSIRO OCE postdoctoral fellowship We thank R. Shivas for the F. oxysporum (strain Fo5176); J. Hane for assisting with Fo5176 genome submission to GenBank; P. Houterman for the SIX4 pRW1p vector; A. Rusu, A. Kettle, and H. Zhao for technical assistance in $F$. oxysporum inoculation experiments; and B. Kidd for critical reading of the manuscript and useful discussions.

\section{LITERATURE CITED}

Altschul, S. F., Madden, T. L., Schäffer, A. A., Zhang, J., Zhang, Z., Miller, W., and Lipman D. J. 1997. Gapped BLAST and PSI-BLAST: A new generation of protein database search programs. Nucleic Acids Res. 25:3389-3402.

Altschul, S. F., Wootton, J. C., Gertz, E. M., Agarwala, R., Morgulis, A., Schäffer, A. A., and Yu, Y. K. 2005. Protein database searches using compositionally adjusted substitution matrices. FEBS (Fed. Eur. Bio- 
chem. Soc.) J. 272:5101-5109.

Anderson, J. P., Badruzsaufari, E., Schenk, P. M., Manners, J. M., Desmond, O. J., Ehlert, C., Maclean, D. J., Ebert, P. R., and Kazan, K. 2004. Antagonistic interaction between abscisic acid and jasmonate-ethylene signaling pathways modulates defense gene expression and disease resistance in Arabidopsis. Plant Cell 16:3460-3479.

Anderson, J. P., Gleason, C. A., Foley, R. C., Thrall, P. H., Burdon, J. B., and Singh, K. B. 2010. Plants versus pathogens: An evolutionary arms race. Funct. Plant Biol. 37:499-512.

Bent, A. F., and Mackey, D. 2007. Elicitors, effectors, and $R$ genes: The new paradigm and a lifetime supply of questions. Annu. Rev. Phytopathol. 45:399-436.

Berrocal-Lobo, M., and Molina, A. 2004. Ethylene response factor 1 mediates Arabidopsis resistance to the soilborne fungus Fusarium oxysporum. Mol. Plant-Microbe Interact. 17:763-770.

Boller, T., and He, S. Y. 2009. Innate immunity in plants: An arms race between pattern recognition receptors in plants and effectors in microbial pathogens. Science 324:742-744.

Campbell, E. J., Schenk, P. M., Kazan, K., Penninckx, I. A., Anderson, J. P., Maclean, D. J., Cammue, B. P., Ebert, P. R., and Manners, J. M. 2003. Pathogen-responsive expression of a putative ATP-binding cassette transporter gene conferring resistance to the diterpenoid sclareol is regulated by multiple defense signaling pathways in Arabidopsis. Plant Physiol. 133:1272-1284.

Chakrabarti, A., Rep, M., Wang, B., Ashton, A., Dodds, P., and Ellis, J. 2011. Variation in potential effector genes distinguishing Australian and non-Australian isolates of the cotton wilt pathogen Fusarium oxysporum f. sp. vasinfectum. Plant Pathol. 60:232-243.

Chisholm, S. T., Coaker, G., Day, B., and Staskawicz, B. J. 2006. Hostmicrobe interactions: Shaping the evolution of the plant innate response. Cell 124:803-814.

Cui, H., Wang, Y., Xue, L., Chu, J., Yan, C., Fu, J., Chen, M., Innes, R. W., and Zhou, J. M. 2010. Pseudomonas syringae effector protein AvrB perturbs Arabidopsis hormone signaling by activating MAP kinase 4 . Cell Host Microbe 7:164-175.

Czymmek, K. J., Fogg, M., Powell, D. H., Sweigard, J., Park, S. Y., and Kang, S. 2007. In vivo time-lapse documentation using confocal and multi-photon microscopy reveals the mechanisms of invasion into the Arabidopsis root vascular system by Fusarium oxysporum. Fungal Genet. Biol. 44:1011-1023.

Diener, A. C., and Ausubel, F. M. 2005. RESISTANCE TO FUSARIUM OXYSPORUM 1, a dominant Arabidopsis disease-resistance gene, is not race specific. Genetics 171:305-321.

Edgar, C. I., McGrath, K. C., Dombrecht, B., Manners, J. M., Maclean, D. C., Schenk, P. M., and Kazan, K. 2006. Salicylic acid mediates resistance to the vascular wilt pathogen Fusarium oxysporum in the model host Arabidopsis thaliana. Australas. Plant Pathol. 35:581-591.

Epple, P., Apel, K., and Bohlmann, H. 1997. Overexpression of an endogenous thionin enhances resistance of Arabidopsis against Fusarium oxysporum. Plant Cell 9:509-520.

Gardiner, D. M., Jarvis, R. S., and Howlett, B. J. 2005. The ABC transporter gene in the sirodesmin biosynthetic gene cluster of Leptosphaeria maculans is not essential for sirodesmin production but facilitates self-protection. Fungal Genet. Biol. 42:257-263.

Gardiner, D. M., Kazan, K., and Manners, J. M. 2009. Novel genes of Fusarium graminearum that negatively regulate deoxynivalenol production and virulence. Mol. Plant-Microbe Interact. 22:1588-1600.

Gohre, V., and Robatzek, S. 2008. Breaking the barriers: Microbial effector molecules subvert plant immunity. Annu. Rev. Phytopathol. 46:189-215.

Houterman, P. M., Speijer, D., Dekker, H. L., de Koster, C. G., Cornelissen, B. J. C., and Rep, M. 2007. The mixed xylem sap proteome of Fusarium oxysporum-infected tomato plants. Mol. Plant Pathol. 8:215-221.

Houterman, P. M., Cornelissen, B. J. C., and Rep, M. 2008. Suppression of plant resistance gene-based immunity by a fungal effector. PLoS Pathog. 4:e1000061. Published online.

Houterman, P. M., Ma, L., van Ooijen, G., de Vroomen, M. J., Cornelissen, B. J., Takken, F. L., and Rep, M. 2009. The effector protein Avr2 of the xylem-colonizing fungus Fusarium oxysporum activates the tomato resistance protein I-2 intracellularly. Plant J. 58:970-978.

Jones, J. D., and Dangl, J. L. 2006. The plant immune system. Nature 444:323-329

Kidd, B. N., Edgar, C. I., Kumar, K. K., Aitken E. A., Schenk, P. M., Manners, J. M., and Kazan, K. 2009. The Mediator complex subunit PFT1 is a key regulator of jasmonate-dependent defense in Arabidopsis. Plant Cell 21:2237-2252.

Kidd, B. N., Kadoo, N. Y., Dombrecht, B., Tekeoglu, M., Gardiner, D. M., Thatcher, L. F., Aitken, E. A., Schenk, P. M., Manners, J. M., and Kazan, K. 2011. Auxin signaling and transport promote susceptibility to the root-infecting fungal pathogen Fusarium oxysporum in Arabidopsis. Mol. Plant-Microbe Interact. 24:733-748.

Lievens, B., Houterman, P. M., and Rep, M. 2009. Effector gene screening allows unambiguous identification of Fusarium oxysporum f. sp. lycopersici races and discrimination from other formae speciales. FEMS (Fed. Eur. Microbiol. Soc.) Microbiol. Lett. 300:201-215.

Ma, L. J., van der Does, H. C., Borkovich, K. A., Coleman, J. J., Daboussi, M. J., Di Pietro, A., Dufresne, M., Freitag, M., Grabherr, M., Henrissat, B., Houterman, P. M., Kang, S., Shim, W. B., Woloshuk, C., Xie, X., Xu, J. R., Antoniw, J., Baker, S. E., Bluhm, B. H., Breakspear, A., Brown, D. W., Butchko, R. A., Chapman, S., Coulson, R., Coutinho, P. M., Danchin, E. G., Diener, A., Gale, L. R., Gardiner, D. M., Goff, S., Hammond-Kosack, K. E., Hilburn, K., Hua-Van, A., Jonkers, W., Kazan, K., Kodira, C. D., Koehrsen, M., Kumar, L., Lee, Y. H., Li, L., Manners, J. M., Miranda-Saavedra, D., Mukherjee, M., Park, G., Park, J., Park, S. Y., Proctor, R. H., Regev, A., Ruiz-Roldan, M. C., Sain, D., Sakthikumar, S., Sykes, S., Schwartz, D. C., Turgeon, B. G., Wapinski, I., Yoder, O., Young, S., Zeng, Q., Zhou, S., Galagan, J., Cuomo, C. A., Kistler, H. C., and Rep, M. 2010. Comparative genomics reveals mobile pathogenicity chromosomes in Fusarium. Nature 464:367-373.

McGrath, K. C., Dombrecht, B., Manners, J. M., Schenk, P. M., Edgar, C. I., Maclean, D. J., Scheible, W., Udvardi, M. K., and Kazan, K. 2005. Repressor- and activator-type ethylene response factors functioning in jasmonate signaling and disease resistance identified via a genomewide screen of Arabidopsis transcription factor gene expression. Plant Physiol. 139:949-959.

Mehrabi, R., Bahkali, A. H., Abd-Elsalam, K. A., Moslem, M., Ben M'barek, S., Gohari, A. M., Jashni, M. K., Stergiopoulos, I., Kema, G. H., and de Wit, P. J. 2011. Horizontal gene and chromosome transfer in plant pathogenic fungi affecting host range. FEMS (Fed. Eur. Microbiol. Soc.) Microbiol. Rev. 35:542-554.

Mes, J. J., Weststeijn, E. A., Herlaar, F., Lambalk, J. J. M., Wijbrandi, J., Haring, M. A., and Cornelissen, B. J. C. 1999. Biological and molecular characterization of Fusarium oxysporum f. sp. lycopersici divides race 1 isolates into separate virulence groups. Phytopathology 89:156-160.

Mes, J. J., van Doorn, A. A., Wijbrandi, J., Simons, G., Cornelissen, B. J., and Haring, M. A. 2000. Expression of the Fusarium resistance gene I-2 colocalizes with the site of fungal containment. Plant J. 23:183-193.

Michielse, C. B, and Rep, M. 2009. Pathogen profile update: Fusarium oxysporum. Mol. Plant Pathol. 10:311-324.

Rep, M., Dekker, H. L., Vossen, J. H., de Boer, A. D., Houterman, P. M., Speijer, D., Back, J. W., de Koster, C. G., and Cornelissen, B. J. 2002. Mass spectrometric identification of isoforms of PR proteins in xylem sap of fungus-infected tomato. Plant Physiol. 130:904-917.

Rep, M., van der Does, H. C., Meijer, M., van Wijk, R., Houterman, P. M. Dekker, H. L., de Koster, C. G., and Cornelissen, B. J. C. 2004. A small, cysteine-rich protein secreted by Fusarium oxysporum during colonization of xylem vessels is required for $I-3$-mediated resistance in tomato. Mol. Microbiol. 53:1373-1383.

Rep, M., Meijer, M., Houterman, P. M., van der Does, H. C., and Cornelissen, B. J. C. 2005. Fusarium oxysporum evades I-3-mediated resistance without altering the matching avirulence gene. Mol. PlantMicrobe Interact. 18:15-23.

Salamov, A. A., and Solovyev, V. V. 2000. Ab initio gene finding in Drosophila genomic DNA. Genome Res. 10:516-522.

Simons, G., Groenendijk, J., Wijbrandi, J., Reijans, M., Groenen, J., Diergaarde, P., Van der Lee, T., Bleeker, M., Onstenk, J., de Both, M. Haring, M., Mes, J., Cornelissen, B., Zabeau, M., and Vos, P. 1998. Dissection of the Fusarium 12 gene cluster in tomato reveals six homologs and one active gene copy. Plant Cell 10:1055-1068.

Stergiopoulos, I., and de Wit, P. J. 2009. Fungal effector proteins. Annu. Rev. Phytopathol. 47:233-263.

Takken F., and Rep, M. 2010. The arms race between tomato and Fusarium oxysporum. Mol. Plant Pathol. 11:309-314.

Thatcher, L. F., Manners, J. M., and Kazan, K. 2009. Fusarium oxysporum hijacks COI1-regulated jasmonate signaling to promote disease in Arabidopsis. Plant J. 58:927-939.

van der Does, H. C., Duyvesteijn, R. G., Goltstein, P. M., van Schie, C. C., Manders, E. M., Cornelissen, B. J., and Rep, M. 2008. Expression of effector gene SIXI of Fusarium oxysporum requires living plant cells. Fungal Genet. Biol. 45:1257-1264.

Van De Wouw, A. P., and Howlett, B. J. 2011. Fungal pathogenicity genes in the age of 'omics'. Mol. Plant Pathol. 12:507-514.

\section{AUTHOR-RECOMMENDED INTERNET RESOURCE}

Softberry software: www.softberry.com 